\title{
Arthroscopic Excision of an Intra-articular Osteoid Osteoma in the Trochlear Notch of Ulna - A Case Report
}

\author{
Rajsirish Bellal Sridharan ${ }^{1}$, Sridhar Gopal Rajagopalan ${ }^{2}$, Senthilvelan Rajagopalan ${ }^{3}$, Nithin \\ Sundaresan ${ }^{1}$, Boblee James ${ }^{4}$
}

Learning Point of the Article:

Intra-articular osteoid osteoma of elbow is quite rare and poses diagnostic challenge. Arthroscopy provides an efficient and minimally invasive option for their management with early return to activities.

\section{Abstract}

Introduction: Osteoid osteoma (OO) is a benign osteoblastic skeletal lesion commonly affecting the diaphysis of long bones. Intra-articular lesions, which present with atypical symptoms, are uncommon and elbow joint involvement is only rarely reported. Conservative treatment is ineffective and surgical excision or ablation is the therapeutic goal in symptomatic patients. Minimally invasive surgery is gaining popularity as a less morbid alternative in joints as opposed to open surgical excision. Herein, we report the first arthroscopic excision of an intra-articular OO involving the trochlear notch of ulna.

Case Report: A 30-year-old man presented with chronic pain and stiffness of his dominant right elbow limiting his routine activities despite treatment with analgesics. Right elbow joint showed features of moderate effusion and X-ray was normal. Computed tomography revealed an $\mathrm{OO}$ in the trochlear notch of ulna breaching the cortex. He underwent arthroscopic en bloc excision of the lesion followed by decompression of the surrounding sclerotic bone and radiofrequency ablation of the base. At 6th month follow-up, the patient was asymptomatic with full range of motion at elbow.

Conclusion: Periarticular OO is a debilitating illness with atypical clinical and radiological features with no response to medical treatment. Although curative, open surgical excision often adds to the morbidity in the postoperative period. Growing experience in elbow arthroscopy has made minimally invasive surgery a safe low morbid alternative procedure. However, a precise knowledge of the instrumentation and anatomy is essential to avoid incomplete excision and recurrence with arthroscopic approach.

Keywords: Osteoid osteoma, elbow, arthroscopy, stiffness, inflammatory arthritis, non-steroidal anti-inflammatory drugs.

\section{Introduction}

Osteoid osteoma (OO) is a small, discrete, and painful benign osteoblastic tumor that predominantly affects young adults. The term OO was coined by Jaffe in 1935 [1]. They are found in the diaphysis (70\%) of long bones, more commonly tibia and femur [2]. They rarely affect the elbow region. Sub periosteal and cancellous types of $\mathrm{OO}$ which accounts for 5-12\% of cases may have intra-articular extension. These patients present with pain, joint effusion, and stiffness mimicking an inflammatory monoarthritis [3]. Radiographic findings are subtle with varying degree of sclerosis. Herein, we report the first arthroscopic excision of an intra-articular $\mathrm{OO}$ involving the trochlear notch of ulna. The diversity in symptoms and absence of the classical salicylate-responsive night pain resulted in a delayed diagnosis in our patient [4].

\section{Case Report}

A 30-year-old man presented with persistent pain, swelling, and stiffness of the right elbow for 18 months with partial relief of symptoms on non-steroidal anti-inflammatory drugs

symptoms on non-steroidal anti-inflammatory drugs
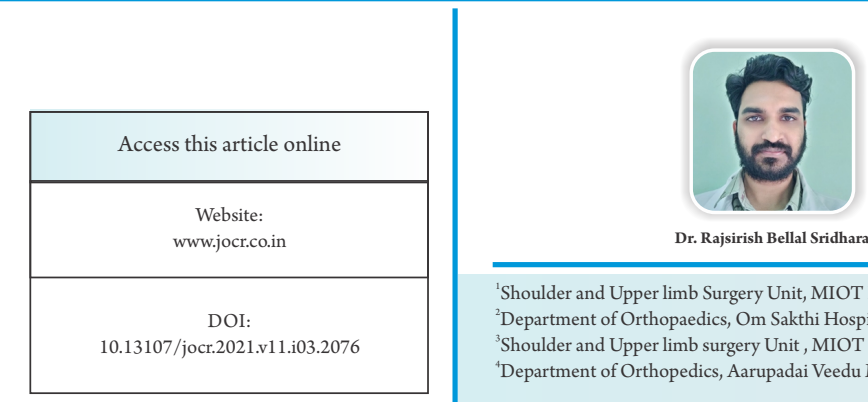

Dr. Rajsirish Bellal Sridharan
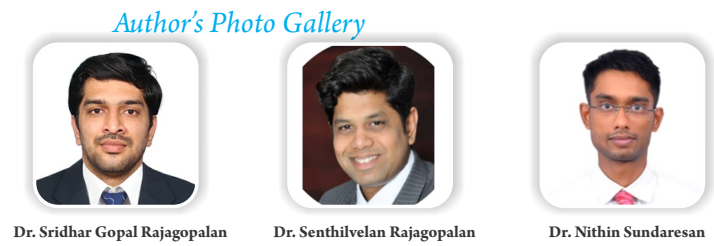

Dr. Nithin Sundaresan

\footnotetext{
Shoulder and Upper limb Surgery Unit, MIOT International, Chennai, Tamil Nadu, India, 2'Department of Orthopaedics, Om Sakthi Hospital, Krishnagiri, Tamil Nadu, India,

${ }^{3}$ Shoulder and Upper limb surgery Unit, MIOT International, Chennai, Tamil Nadu, India,

"Department of Orthopedics, Aarupadai Veedu Medical College and Hospital, Pondicherry, India.

Address of Correspondence:

Dr. Nithin Sundaresan

Department of Orthopaedics, SRM Medical College and Research Centre, Chennai, Tamil Nadu, India.

E-mail: nithin2441992@gmail.com
}

Journal of Orthopaedic Case Reports | pISSN 2250-0685 | eISSN 2321-3817 | Available on www.jocr.co.in | doi:10.13107/jocr.2021.v11.i03.2076 This is an Open Access article distributed under the terms of the Creative Commons Attribution Non-Commercial License (http://creativecommons.org/licenses/by-nc/3.0) which permits unrestricted non-commercial use, distribution, and reproduction in any medium, provided the original work is properly cited. 


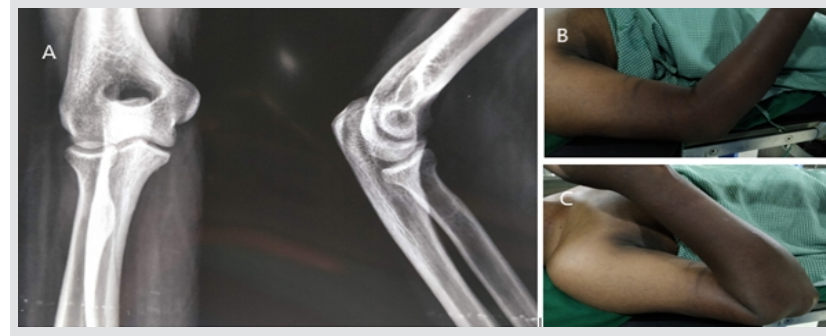

Figure 1: (a) Initial radiograph showed no striking abnormalities (b and c). The patient had passive range of motion: $30-140^{\circ}$ limited by pain and stiffness.

(NSAIDs) and disease modifying anti rheumatoid drugs. No fever, night pain, or any other joint involvement was noted and there was no history of any trauma. On physical examination, there was moderate effusion, $20-110^{\circ}$ of flexion-extension, normal pronosupination, and a stable elbow joint (Fig. 1).X-ray was normal and inflammatory markers were all negative. Synovial fluid analysis suggested non-specific inflammation and culture was sterile. Computed tomography (CT) evaluation revealed a well-defined lucent nidus with a central sclerotic focus $(5 \mathrm{~mm})$ breaching the articular margins of trochlear notch with limited sclerosis in the periphery (Fig. 2a, b). There was also reactive fluffy periosteal reaction and edema involving adjacent intermuscular plane of proximal forearm. Magnetic resonance imaging (MRI) showed a hypointense central nidus in the trochlear notch on $\mathrm{T} 1$ and $\mathrm{T} 2$ sequences with minimal $\mathrm{T} 2$ hyperintensity in the periphery suggesting unmineralized stroma. A surrounding hypointense sclerosis was visualized in T1 sequence. There was also bone edema involving the proximal ulna with minimal joint effusion and synovial proliferation of the elbow joint (Fig. $2 \mathrm{c}$ and d).

With a provisional diagnosis of $\mathrm{OO}$, we planned a diagnostic arthroscopy of the right elbow to look for arthroscopic excision and consent was obtained for open excision if access appeared difficult.

Under general anesthesia, the patient was positioned in lateral decubitus, arm suspended in an elbow support, and a proximal tourniquet placed. Insufflation with $20 \mathrm{ml}$ saline through the soft spot portal helped in distension of capsule and pushing away of the major neurovascular structures from the field. Diagnostic arthroscopy was done using a $2.8 \mathrm{~mm} 30^{\circ}$ arthroscope through direct lateral (soft spot) portal (Fig. 3a, b, d). There was florid synovial reaction in the ulnohumeral joint overlying a purplish red area in the trochlear notch (Fig. $3 \mathrm{c}$ and e). An inside out proximal anteromedial working portal was made $1 \mathrm{~cm}$ proximal and in front of the medial epicondyle. Blunt probe palpation revealed a soft and easily yielding lesion with surrounding dense unyielding trabecular bone. Synovial tissue was sampled for evaluation using cupped biter. An accessory posterolateral portal was capitellar and radioulnar joints, and (e) synovitis in the medial joint space.

\section{Discussion}

$\mathrm{OO}$ is a benign lesion comprising $10-12 \%$ of bone tumors [5] with a male predominance mostly in their second and third decade $[3,6]$. The more common diaphyseal tumors present with classical night pain relieved by salicylate/NSAIDs and characteristic X-ray finding of a radiolucent nidus with typical surrounding profound sclerosis $[7,8]$. High levels of prostaglandin E2 in the nidus have been attributed to the classical night pain which relieves on prostaglandin inhibitors [6]. Surgical excision gives best results in symptomatic patients, while percutaneous ablation is a safe alternative. On the other hand, intra-articular $\mathrm{OO}$ is an uncommon entity and elbow is very rare site of occurrence, wherein olecranon fossa, trochlea or capitulum, proximal radial, proximal ulna, coronoid fossa,
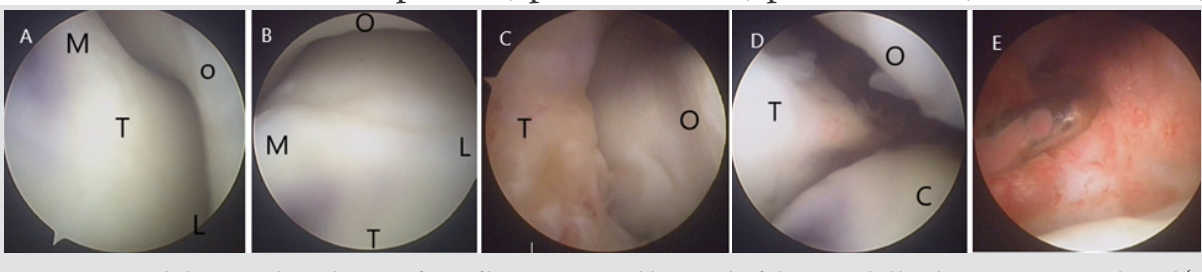

Figure 3: M: Medial L: Lateral O: Olecranon fossa of humerus T: Trochlear notch of ulna R: Radial head. Diagnostic scopy showed (a and b) normal ulnohumeral articulation, (c) fluffy synovial reaction posteriorly overlying the osteoid osteoma, (d) normal radio- 


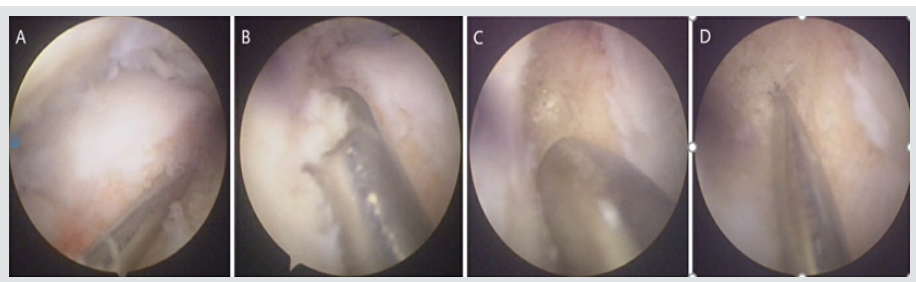

Figure 4: (a) Lesion excised with an arthroscopic chisel (b) surrounding sclerotic bone removed with a ring curette, arthroscopic burr, (c and d) sclerotic base of the lesion was decompressed using k-wire, and base was ablated using a radiofrequency probe.

and medial epicondyle can all be involved [8]. In our case, the lesion was found in the proximal ulna breaching the articular cartilage of trochlear notch.

The classical clinical picture of salicylate-responsive night pain may not be present in intra-articular OOs [9]. Most cases present like inflammatory pathologies of a single joint with symptoms such as pain, swelling, and stiffness [10]. Synovitis is responsible for the effusion that restricts ROM in these cases [7]. Differential diagnosis includes inflammatory and infectious monoarticular arthritis such as rheumatoid and tubercular arthritis [8]. X-ray may not show the classical osteoblastic reaction in intra-articular varieties as there is little or no reactive sclerosis [8]. Moreover, the nidus can show varying degree of maturity and sclerosis causing diagnostic dilemma in most cases [11]. This atypical clinical and imaging finding often results in a delay of around 2 years for a diagnosis to be made $[3,4,7]$.

CT imaging is considered most specific [3] and superior to MRI scan [6]. MRI can show the nidus but defines the soft-tissue features such as joint effusion and synovial pathology better resulting in diagnostic confusions unless interpreted with caution [6]. Technicium-99 bone scintigraphy shows a hot spot surrounding an area of inactivity but may be absent in joint pathology due to surrounding synovial proliferation and hyperemia. Histopathology showing irregular immature woven bone trabeculae covered by activated osteoblasts embedded in vascular fibrous stroma with scattered osteoclasts provides the final diagnosis [3].

Treatment modalities include percutaneous CT-guided excision techniques, percutaneous radiofrequency ablation, and open or arthroscopic excision [8]. Intra-articular radiofrequency ablation can cause osteonecrosis or cartilage damage $[3,8]$, while open surgery is associated with longer post-operative morbidity. Recently, various authors have reported arthroscopic excision of the lesion as a less invasive approach [12]. The direct magnified vision under arthroscope, the option to perform further maneuverers such as synovial biopsy/debridement and capsular releases to improve ROM are additional advantages. Capsular release was not required in our case, where mobility was not restricted. Bhatia in his article has provided detailed 7 steps and technical tips for easy identification and excision of the lesion [13]. Kelly et al. describe the technique of using a ring curette to remove the nidus and burr to extend the curettage to the peripheral sclerotic bone [14]. Better convalescence with drastic pain relief and early mobilization is a definite advantage of arthroscopic excision. However, large and inaccessible lesions pose the risk of incomplete excision. Nourissat et al. reported a case of OO of distal humerus treated arthroscopically, who presented with persistent pain 6 weeks after surgery. A repeat CT in this case showed incomplete excision warranting a second surgery [15]. Radiofrequency ablation should also be used with caution in a joint due to unpredictability of the limit of osteonecrosis [8].

\section{Conclusion}

Intra-articular $\mathrm{OO}$ is a rare cause of unresolving monoarthritic type of elbow pain and elbow joint is a possible site. Once diagnosed with CT scan, elbow arthroscopy is a minimally invasive approach providing good vision for excision, decompression, and radiofrequency ablation of the base with a word of caution against inaccessibility, incomplete removal, and recurrence in inexperienced hands and large lesions.

\section{Clinical Message}

The study outlines that intra-articular OO of elbow joint, though rare, should be considered in differential diagnosis of prolonged monoarthritic pain in large and small joints including elbow. A CT scan is essential for diagnosis and localization of the lesion. As opposed to arthrotomy, arthroscopic removal of intra-articular $\mathrm{OO}$ provides excellent relief of symptoms and early post-operative recovery with a possible risk of incomplete removal in difficult situations. 


\section{References}

1. Jaffe HL. Osteoid osteoma. A benign osteoblastic tumor composed of osteoid and atypical bone. Arch Surg 1935;31:709-28.

2. Campanacci M, Ruggieri P, Gasbarrini A, Ferraro A, Campanacci L. Osteoid osteoma. Direct visual identification and intralesional excision of the nidus with minimal removal of bone. J Bone Joint Surg Br 1999;81:814-20.

3. Rolvien T, Zustin J, Mussawy H, Schmidt T, Pogoda P, Ueblacker P. Intra-articular osteoid osteoma as a differential diagnosis of diffuse monoarticular joint pain. BMC Musculoskelet Disord 2016;17;455.

4. Ditsios K, Papadakis N, Theodoroudis I, Kostretzis L, Konstantinou P, Pinto I, et al. A misguiding osteoid osteoma in the bicipital tuberosity of the radius. Case Rep Orthop 2016;2016:6428137.

5. Dahlin DC, Unni KK. Bone Tumors: General Aspects and Data on 8,542 Cases. 4th ed. Springfield, IL: Thomas; 1987.

6. Kraus G, Fortes S, Vazquez J, Renfree KJ. Intra-articular osteoid osteoma of the proximal ulna. Orthopedics 2014;37:e214-7.

7. Kim JH, Lee HJ, Baek GH. Intra-articular osteoid osteoma in the proximal ulna combined with radial head subluxation: A case report.J Shoulder Elbow Surg 2012;21:e1-5.
8. Goyal T, Pandita N, Paul S. Arthroscopic excision of intraarticular sub-periosteal osteoid osteoma of elbow: A case report. SICOTJ 2018;4:25.

9. Kniesl JS, Simon MA. Medical management compared with operative treatment for osteoid osteoma. J Bone Joint Surg Am 1992;74:179-85.

10. Weber KL, Morrey BF. Osteoid osteoma of the elbow: A diagnostic challenge. J Bone Joint Surg Am 1999;81:11119.

11. Cristina CA, de Melo RT, de Castro RC, Souza F. Diagnostic difficulties in Osteoid osteoma of the elbow: Clinical, radiological and histopathological study. Radiol Bras 2012;45:13-9.

12. Hatta T, Hosaka M, Watanuki M, Yano T, Yoshida S, Watanabe $\mathrm{M}$, et al. Arthroscopic excision of intra-articular osteoid osteoma at the elbow. Case Rep Orthop 2019;2019:8505382.

13. Bhatia DN. Arthroscopic excision of osteoid osteoma of the elbow. Arthrosc Tech 2017;6:e543-8.

14. Kelly AM, Selby RM, Lumsden E, O’Brien SJ, Drakos MC. Arthroscopic removal of an osteoid osteoma of the shoulder. Arthroscopy 2002;18:801-6.

15. Nourissat G, Kakuda C, Dumontier C. Arthroscopic excision of osteoid osteoma of the elbow. Arthroscopy 2007;23:799.e1-4.
Conflict of Interest: Nil

Source of Support: Nil

Consent: The authors confirm that informed consent was obtained from the patient for publication of this case report

\section{How to Cite this Article}

Rajsirish BS, Senthilvelan R, Rajagopalan S, Sundaresan N, James B. Arthroscopic excision of an intra-articular osteoid osteoma in the trochlear notch of ulna - A case report. Journal of Orthopaedic Case Reports 2021 March;11(3):33-36. 